УДК 517.53

\title{
Coefficient Multipliers for the Privalov class in a Disk
}

\author{
Eugenia G. Rodikova* \\ Bryansk State University
}

Bezhitskaya, 14, Bryansk, 241036

Russia

Received 02.05.2018, received in revised form 20.07.2018, accepted 06.09.2018

We obtain exact estimates of the growth and the Taylor coefficients of analytic functions from the Privalov classes in the unit disk. Also we describe coefficient multipliers from the Privalov classes into the Hardy classes.

Keywords: Privalov classes, Taylor coefficients, coefficient multiplier, maximal growth, analytic functions.

DOI: $10.17516 / 1997-1397-2017-11-6-723-732$.

\section{Introduction}

Let $\mathbb{C}$ be the complex plane, $D$ be the unit disk on $\mathbb{C}, H(D)$ be the set of all functions, holomorphic in $D$. For all $0<q<+\infty$ we define the Privalov class of function $\Pi_{q}$ as follows (see [8]):

$$
\Pi_{q}=\left\{f \in H(D): \sup _{0<r<1} \frac{1}{2 \pi} \int_{-\pi}^{\pi}\left(\ln ^{+}\left|f\left(r e^{i \theta}\right)\right|\right)^{q} d \theta<+\infty\right\} .
$$

Note that the classes $\Pi_{q}$ were first considered by I. I. Privalov in [8]. In the case $1 \leqslant q<+\infty$ the Privalov spaces were studied by M.Stoll, V.I. Gavrilov, A.V.Subbotin, D. A. Efimov, R. Mestrovic, Z. Pavicevic, etc. The monograph [2] contains a brief overview of their results. The case $0<q<1$ has been little studied in the scientific literature. In this paper we obtain exact estimates for the maximum modulus and the Taylor coefficients of functions from the classes $\Pi_{q}(0<q<1)$ (Section 1), on this basis we describe coefficient multipliers from the Privalov classes $\Pi_{q}(0<q<1)$ into the Hardy classes $H^{p}(0<p<+\infty)$ (Section 2).

Notice that the problem of describing the Taylor coefficients for analytic functions of the Nevanlinna class was first solved by S. N. Mergelyan in the early 20th century (see [9, p. 152]). Later on these questions in the Hardy classes were investigated by G. Hardy and D. Littlewood, A. A. Friedman (see [3]), in V. I. Smirnov's classes by N. Yanagihara [20], in the Privalov classes $\Pi_{q}(q>1)$ by M.Stoll [18], in the plane Nevanlinna classes by S. V. Shvedenko [17], in the weighted classes of analytic functions in a disk with restrictions on the Nevanlinna characteristic by F. A. Shamoyan and E. N. Shubabko [14], and by the author of this paper (see [10]).

As the authors observe in [2, p. 148], the notion of a coefficient multiplier arises naturally in the study of asymptotic properties of the Taylor coefficients for functions from certain classes. In a simplified form, the problem is posed as follows: which factors the Taylor coefficients of a function of a given class must be multiplied by for them to acquire special properties, for example, being bounded or form an absolutely convergent series. Requiring that the resulting

*evheny@yandex.ru

C) Siberian Federal University. All rights reserved 
products be Taylor coefficients of functions from some other class, we arrive to definition of the coefficient multiplier.

Let $X$ and $Y$ be some classes of functions analytic in the unit disk $D$.

Definition 1. The sequence of complex numbers $\Lambda=\left\{\lambda_{k}\right\}_{k=1}^{+\infty}$ is called a coefficient multiplier from class $X$ into class $Y$ if for any function $f \in X, f(z)=\sum_{k=0}^{+\infty} a_{k} z^{k}$, we have $\Lambda(f)(z)=$ $=\sum_{k=0}^{+\infty} \lambda_{k} a_{k} z^{k} \in Y$. It is denoted by $C M(X, Y)$.

Numerous works are devoted to the description of multipliers in various classes of holomorphic functions. We recall some of them: $[1,2,4-6,11,13-16,19]$, and etc.

\section{On maximal growth and the Taylor coefficients for functions from the Privalov classes}

The following statement is valid:

Theorem 1.1. If $f \in \Pi_{q}$ then

$$
\ln ^{+} M(r, f)=o\left((1-r)^{-1 / q}\right), r \rightarrow 1-0,
$$

where $M(r, f)=\max _{|z|=r}|f(z)|$.

Remark. Throughout the paper we study the Privalov class $\Pi_{q}$ with the parameter $0<q<1$. Also, unless otherwise stated, we denote by $c, c_{1}, \ldots, c_{n}(\alpha, \beta, \ldots)$ arbitrary positive constants depending on $\alpha, \beta, \ldots$, whose specific values are immaterial.

Proof. We choose an arbitrary point $z_{0} \in D$ and by definition put $K_{z_{0}}=\left\{\zeta \in D:\left|\zeta-z_{0}\right|<\right.$ $\left.\frac{1}{2}\left(1-\left|z_{0}\right|\right)\right\}$. Let $d m_{2}$ be the planar Lebesgue measure. From the inequality (see [7, p. 144], Theorem 9.1.1, equation(9.3)) which holds for all $0<q<1$ :

$$
\left(\ln ^{+}\left|f\left(z_{0}\right)\right|\right)^{q} \leqslant \frac{c_{q}}{\left(1-\left|z_{0}\right|\right)^{2}} \int_{K_{z_{0}}}\left(\ln ^{+}|f(\zeta)|\right)^{q} d m_{2}(\zeta)
$$

we obtain

$$
\left(\ln ^{+}\left|f\left(z_{0}\right)\right|\right)^{q} \leqslant \frac{c_{q}}{\left(1-\left|z_{0}\right|\right)^{2}} \int_{\left|z_{0}\right|-\frac{1-\left|z_{0}\right|}{2}}^{\left|z_{0}\right|+\frac{1-\left|z_{0}\right|}{2}} \int_{-\pi}^{\pi}\left(\ln ^{+}\left|f\left(\rho e^{i \theta}\right)\right|\right)^{q} d \theta d \rho,
$$

whence we have:

$$
\begin{aligned}
\left(\ln ^{+}\left|f\left(z_{0}\right)\right|\right)^{q} \leqslant \frac{c_{q}}{\left(1-\left|z_{0}\right|\right)} \sup _{\left|z_{0}\right|-\frac{1-\left|z_{0}\right|}{2}<\rho<\left|z_{0}\right|+\frac{1-\left|z_{0}\right|}{2}} \int_{-\pi}^{\pi}\left(\ln ^{+}\left|f\left(\rho e^{i \theta}\right)\right|\right)^{q} d \theta \leqslant \\
\leqslant \frac{c_{q}}{\left(1-\left|z_{0}\right|\right)} \sup _{0<\rho<1} \int_{-\pi}^{\pi}\left(\ln ^{+}\left|f\left(\rho e^{i \theta}\right)\right|\right)^{q} d \theta
\end{aligned}
$$

Now the required estimate (1) follows. 
Theorem 1.2. If $\sum_{k=0}^{+\infty} a_{k} z^{k}$ is the Taylor series expansion for a function $f \in \Pi_{q}$, then

$$
\ln ^{+}\left|a_{k}\right|=o\left(k^{\frac{1}{1+q}}\right), k \rightarrow+\infty .
$$

Proof. We prove this theorem using the method of S. N. Mergelyan (see [9, p. 152]). From the Cauchy inequality and the estimate (1) of Theorem 1.1 it follows that for any arbitrarily small $\varepsilon>0$ there exists $r_{\varepsilon} \in(0,1)$ such that

$$
\left|a_{k}\right| \leqslant r^{-k} \exp \left\{\varepsilon(1-r)^{-\frac{1}{q}}\right\}, r_{\varepsilon}<r<1, n=0,1, \ldots,
$$

which is equivalent to

$$
\ln ^{+}\left|a_{k}\right| \leqslant \varepsilon(1-r)^{-\frac{1}{q}}-k \ln r, r_{\varepsilon}<r<1, n=0,1, \ldots .
$$

Introduce the function

$$
\phi(r)=\varepsilon(1-r)^{-\frac{1}{q}}-k \ln r .
$$

We investigate it to find its exact lower bound. Calculate the derivative:

$$
\phi^{\prime}(r)=\frac{\varepsilon}{q} \cdot \frac{1}{(1-r)^{\frac{1}{q}+1}}-\frac{k}{r} .
$$

We find the minimum of the function $\phi(r)$, solving the equation $\phi^{\prime}(r)=0$ :

$$
\frac{\varepsilon}{q} \cdot \frac{r}{(1-r)^{\frac{1}{q}+1}}=k .
$$

The solution of this equation exists and unique on the interval $(0,1)$.

For convenience, we introduce the following notation:

$$
c_{k}=\frac{1}{\delta \sqrt{r_{k}}}, \quad s_{k}=\frac{1-r_{k}}{\delta \sqrt{r_{k}}},
$$

where $\delta>1$.

We can assume that $s_{k}<c_{k} \leqslant 1$. Indeed, the inequality $s_{k}<c_{k}$ is obvious.

Now, $c_{k} \leqslant 1$ is equivalent to

$$
\sqrt{r_{k}} \geqslant \frac{1}{\delta}
$$

while $s_{k}<1$ is equivalent to

$$
\sqrt{r_{k}}>\frac{\sqrt{\delta^{2}+4}-\delta}{2}
$$

and (6) follows from (7).

In the new notation equation (5) takes the form:

$$
\frac{\varepsilon}{q \delta^{2}} \cdot \frac{1}{s_{k}^{2}} \cdot\left(\frac{c_{k}}{s_{k}}\right)^{\frac{1}{q}-1}=k
$$

or

$$
\frac{s_{k}^{\frac{1}{q}+1}}{c_{k}^{\frac{1}{q}-1}}=\frac{\varepsilon}{k q \delta^{2}} .
$$

Since $c_{k} \leqslant 1$, the last equality implies the estimate:

$$
s_{k} \leqslant\left(\frac{\varepsilon}{k q \delta^{2}}\right)^{\frac{q}{q+1}}
$$


From the same equation we obtain:

$$
\left(\frac{c_{k}}{s_{k}}\right)^{\frac{1}{q}}=\left(\frac{k s_{k}^{2} q \delta^{2}}{\varepsilon}\right)^{\frac{1}{1-q}}
$$

Taking into account the estimate (8), we have:

$$
\left(\frac{c_{k}}{s_{k}}\right)^{\frac{1}{q}} \leqslant\left(\frac{q \delta^{2}}{\varepsilon}\right)^{\frac{1}{1+q}} \cdot k^{\frac{1}{1+q}}
$$

Using (8), (9), we estimate the value of the function $\phi(r)$ at the minimum point $r=r_{k}$ :

$$
\phi\left(r_{k}\right)=\varepsilon\left(1-r_{k}\right)^{-\frac{1}{q}}-k \ln r_{k}
$$

The application of (9) yields:

$$
\phi\left(r_{k}\right) \leqslant \varepsilon\left(\frac{q \delta^{2}}{\varepsilon}\right)^{\frac{1}{1+q}} \cdot k^{\frac{1}{1+q}}-k \ln r_{k}
$$

To estimate the last term we note that

$$
\frac{\left(r_{k}\right)^{-\frac{1}{2}}-r_{k}^{\frac{1}{2}}}{2}=\frac{\exp \left(-\frac{1}{2} \ln r_{k}\right)-\exp \left(\frac{1}{2} \ln r_{k}\right)}{2}=-\operatorname{sh}\left(\frac{1}{2} \ln r_{k}\right)=\operatorname{sh}\left(-\frac{1}{2} \ln r_{k}\right)=\frac{s_{k} \delta}{2}
$$

whence

$$
\begin{gathered}
-\ln r_{k}=2 \operatorname{arcsh} \frac{s_{k} \delta}{2} \leqslant 2 \frac{s_{k} \delta}{2} \\
-k \ln r_{k} \leqslant k s_{k} \delta .
\end{gathered}
$$

Thus we have:

$$
\phi\left(r_{k}\right) \leqslant k^{\frac{1}{1+q}} \varepsilon^{\frac{q}{1+q}}\left(q \delta^{2}\right)^{\frac{1}{1+q}} \cdot\left(1+\frac{1}{q \delta}\right) .
$$

The required estimate (2) follows.

\section{Description of the coefficient multipliers from the Privalov classes into the Hardy classes}

Theorem 2.1. Let $0<p \leqslant+\infty, \Lambda=\left\{\lambda_{k}\right\}_{k=1}^{+\infty} \subset \mathbb{C}$. For $\Lambda=C M\left(\Pi_{q}, H^{p}\right)$ it is necessary and sufficient to have

$$
\left|\lambda_{k}\right|=O\left(\exp \left(-c \cdot k^{\frac{1}{q+1}}\right)\right), k \rightarrow+\infty
$$

for some $c>0$.

The proof of this theorem is based on auxiliary statements.

Lemma 2.2. (see [2, Lemma 9.7]) Let $F$ and $H$ be linear classes of holomorphic functions in the unit disk $D$ with metrics, convergence in which is not weaker than the uniform convergence on compact subsets of $D$. Then each coefficient multiplier from class $F$ into class $H$ is a linear and closed operator.

To formulate the next Lemma we introduce a metric on the class $\Pi_{q}$ :

$$
\rho(f, g)=\sup _{0<r<1} \frac{1}{2 \pi} \int_{-\pi}^{\pi} \ln ^{q}\left(1+\left|f\left(r e^{i \theta}\right)-g\left(r e^{i \theta}\right)\right|\right) d \theta .
$$


Lemma 2.3. The class $\Pi_{q}$ with respect to the metric (12) is an F-space.

Proof. The proof of this statement is equivalent to establishing the properties a)-d) of a metric (see [12]):

a) $\rho(f, g)=\rho(f-g, 0)$ is obvious.

b) $\Pi_{q}$ is a complete metric space.

Let $\left\{f_{n}\right\}$ be an arbitrary fundamental (Cauchy) sequence from the class $\Pi_{q}$, i.e. $\forall \varepsilon>0$ $\exists N(\varepsilon)>0: \forall n, m>N \Rightarrow \rho\left(f_{n}, f_{m}\right)<\varepsilon$. We show that it converges to some function $f \in \Pi_{q}$. Note that functions $\ln \left(1+\left|f_{n}\right|\right)$ are subharmonic in $D$. In the same way as in the proof of Theorem 1.1 we use the equation from Theorem 9.1 .1 of $[7$, p. 144] to obtain:

$$
\begin{gathered}
\ln ^{q}\left(1+\left|f_{n}\left(R e^{i \varphi}\right)-f_{m}\left(R e^{i \varphi}\right)\right|\right) \leqslant \frac{c_{q}}{(1-R)^{2}} \sup _{0<r<1} \int_{-\pi}^{\pi} \ln ^{q}\left(1+\left|f_{n}\left(r e^{i \theta}\right)-f_{m}\left(r e^{i \theta}\right)\right|\right) d \theta \\
\ln ^{q}\left(1+\left|f_{n}\left(R e^{i \varphi}\right)-f_{m}\left(R e^{i \varphi}\right)\right|\right) \leqslant \frac{2 \pi c_{q}}{(1-R)^{2}} \rho\left(f_{n}, f_{m}\right),
\end{gathered}
$$

whence we have

$$
\left|f_{n}\left(R e^{i \varphi}\right)-f_{m}\left(R e^{i \varphi}\right)\right| \rightarrow 0, n, m \rightarrow+\infty,
$$

for all $0<R<1, \varphi \in[-\pi, \pi]$. So the sequence $\left\{f_{n}\right\}$ converges uniformly in the unit disk for some function $f \in H(D)$. Now we prove that $f \in \Pi_{q}$.

$$
\begin{gathered}
\sup _{0<r<1} \frac{1}{2 \pi} \int_{-\pi}^{\pi}\left(\ln ^{+}\left|f\left(r e^{i \theta}\right)\right|\right)^{q} d \theta \leqslant \sup _{0<r<1} \frac{1}{2 \pi} \int_{-\pi}^{\pi} \ln \left(1+\left|f\left(r e^{i \theta}\right)\right|\right)^{q} d \theta \leqslant \\
\leqslant \sup _{0<r<1} \frac{1}{2 \pi} \int_{-\pi}^{\pi} \ln ^{q}\left(1+\left|f\left(r e^{i \theta}\right)-f_{n}\left(r e^{i \theta}\right)\right|+\left|f_{n}\left(r e^{i \theta}\right)\right|\right) d \theta .
\end{gathered}
$$

Since $(a+b)^{q} \leqslant\left(a^{q}+b^{q}\right)$ for any $a>0, b>0,0<q<1$, we have

$$
\begin{aligned}
\sup _{0<r<1} \frac{1}{2 \pi} \int_{-\pi}^{\pi} & \left(\ln ^{+}\left|f\left(r e^{i \theta}\right)\right|\right)^{q} d \theta \leqslant \\
& \leqslant \sup _{0<r<1} \frac{1}{2 \pi} \int_{-\pi}^{\pi}\left(\ln ^{q}\left(1+\left|f\left(r e^{i \theta}\right)-f_{n}\left(r e^{i \theta}\right)\right|\right)+\ln ^{q}\left(1+\left|f_{n}\left(r e^{i \theta}\right)\right|\right)\right) d \theta \leqslant \text { const. }
\end{aligned}
$$

We conclude that $\Pi_{q}$ is a complete metric space.

c) If $f, f_{n} \in \Pi_{q}$ and $\rho\left(f_{n}, f\right) \rightarrow 0, n \rightarrow+\infty$, then for any $\beta \in \mathbb{C}$ we have $\rho\left(\beta f_{n}, \beta f\right) \rightarrow 0$, $n \rightarrow+\infty$.

For $|\beta|<1$ the property immediately follows. Assume that $|\beta|>1$, without loss of generality we may take $\beta>1$. Since the sequence $\left\{f_{n}\right\}$ converges, it is fundamental (Cauchy). As stated above, from that follows its uniform convergence inside $D$.

Since for any $\beta \geqslant 1$ and $x \geqslant 0$ the estimate $(1+\beta x) \leqslant(1+x)^{\beta}$ is valid, we have

$$
\begin{aligned}
& \rho\left(\beta f_{n}, \beta f\right)=\sup _{0<r<1} \frac{1}{2 \pi} \int_{-\pi}^{\pi} \ln ^{q}\left(1+\beta\left|f_{n}\left(r e^{i \theta}\right)-f\left(r e^{i \theta}\right)\right|\right) d \theta \leqslant \\
& \leqslant \sup _{0<r<1} \frac{\beta^{q}}{2 \pi} \int_{-\pi}^{\pi} \ln ^{q}\left(1+\left|f_{n}\left(r e^{i \theta}\right)-f\left(r e^{i \theta}\right)\right|\right) d \theta=\beta^{q} \rho\left(f_{n}, f\right),
\end{aligned}
$$

whence the property c) follows.

d) If $\beta_{n}, \beta \in \mathbb{C}$ and $\beta_{n} \rightarrow \beta$, then we have $\rho\left(\beta_{n} f, \beta f\right) \rightarrow 0, n \rightarrow+\infty$ for any function $f \in \Pi_{q}$. The property immediately follows from the inequality

$$
\ln \left(1+\left|\beta_{n}-\beta\right||f|\right) \leqslant \ln (1+|f|)+\ln \left(1+\left|\beta_{n}-\beta\right|\right) .
$$

Lemma 2.3 is proved. 
Lemma 2.4. Let the sequence of complex numbers $\left\{\lambda_{k}\right\}_{k=1}^{+\infty}$ satisfy the condition:

$$
\left|\lambda_{k}\right|=O\left(\exp \left(-c_{k} \cdot k^{\frac{1}{q+1}}\right)\right), k \rightarrow+\infty
$$

for an arbitrary positive sequence $\left\{c_{k}\right\}_{k=1}^{+\infty}, c_{k} \downarrow 0, k \rightarrow+\infty$. Then there exists $c>0$ such that for all $k \in \mathbb{N}$ the condition (11) is true.

The proof of Lemma 2.4 repeats the arguments of the article by N. Yanagihara [19] (see Lemma 1) with the exponent $\frac{1}{q+1}$.

Lemma 2.5. Let $0<q<1$,

$$
g(z)=\exp \frac{c}{(1-z)^{\frac{1}{q}}}, \quad z \in D
$$

where $0<c<\frac{1}{q}, \sum_{n=1}^{+\infty} a_{n}(c) z^{n}$ be the Taylor series expansion for $g$. Then the following estimate is valid:

$$
\left|a_{n}(c)\right| \geqslant \exp \left(c^{\frac{q}{q+1}} \cdot n^{\frac{1}{q+1}}\right) .
$$

The proof of Lemma 2.5 repeats the arguments presented in the author's thesis (see [11, p. 104], Lemma 2.7) with the exponent of $\frac{1}{q}$. The method goes back to Mergelyan S. N. (see [9]).

As stated above, from $\rho\left(f_{n}, f\right) \rightarrow 0, n \rightarrow+\infty$ it follows that the sequence $f_{n}(z)$ uniformly converges to $f(z)$ in $D$. Therefore if $f_{n}(z)=\sum_{k=0}^{+\infty} a_{k}^{(n)} z^{k}$ and $f(z)=\sum_{k=0}^{+\infty} a_{k} z^{k}$, then $a_{k}^{(n)} \rightarrow a_{k}$, $n \rightarrow+\infty$.

Let $X$ be an F-space consisting of complex sequences $\left\{b_{k}\right\}_{k}$ such that convergence of a sequence $\beta^{(n)}=\left\{b_{k}^{(n)}\right\}$ to $\beta=\left\{b_{k}\right\}, n \rightarrow+\infty$ implies coordinate-wise convergence $b_{k}^{(n)} \rightarrow b_{k}$, $n \rightarrow+\infty, k=0,1,2, \ldots$.

Consider a coefficient multiplier $\Lambda=C M\left(\Pi_{q}, X\right)$. By Lemma 2.2, $\Lambda$ is a closed operator, therefore by the closed graph theorem (see [12]) $\Lambda$ is a continuous operator and it maps bounded sets in the class $\Pi_{q}$ into bounded sets in $X$.

Proof of Theorem 2.1. Let $\Lambda=\left\{\lambda_{k}\right\}_{k=1}^{+\infty}$ be a coefficient multiplier from the class $\Pi_{q}$ into the class $X$. We prove that there exists $c>0$ such that the estimate (11) is valid, i.e.

$$
\left|\lambda_{k}\right|=O\left(\exp \left(-c \cdot k^{\frac{1}{q+1}}\right)\right), k \rightarrow+\infty
$$

According to Lemma 2.4, it is enough to show that $\Lambda$ satisfies the estimate (13) for some positive infinitesimal sequence $\left\{c_{k}\right\}$.

We choose the sequence $\left\{c_{k}\right\}$ so that the following estimates are valid:

$$
k^{-\frac{1}{2 q}} \leqslant c_{k} \leqslant \frac{1}{2}
$$

We consider in $\Pi_{q}$ the sequence of functions

$$
f_{k}(z)=g\left(r_{k} z\right)=\exp \frac{c_{k}}{\left(1-r_{k} z\right)^{\frac{1}{q}}}, k=1,2, \ldots, 0<r_{k}<1,
$$

satisfying the conditions of Lemma 2.5, and the double inequality

$$
\sqrt{1-\frac{\pi^{2}}{k-1}} \leqslant r_{k} \leqslant \sqrt{1-\frac{\pi^{2}}{B_{k}-1}}, \quad B_{k}=\pi\left(\frac{\gamma_{k}}{c_{k}}\right)^{q}, \quad B_{k}>1
$$


where $\gamma_{k}$ is a positive infinitesimal sequence such that $\frac{c_{k}}{\gamma_{k}} \leqslant k^{-\frac{1}{q}}, k=1,2, \ldots$.

It is obvious that $r_{k} \rightarrow 1-0, k \rightarrow+\infty$.

Let us show that $f_{k} \in \Pi_{q}$.

$$
\begin{aligned}
& \sup _{0<r<1} \frac{1}{2 \pi} \int_{-\pi}^{\pi}\left(\ln ^{+}\left|f_{k}\left(r e^{i \theta}\right)\right|\right)^{q} d \theta=\sup _{0<r<1} \frac{1}{2 \pi} \int_{-\pi}^{\pi}\left(\ln ^{+}\left|\exp \frac{c_{k}}{\left(1-r_{k} r e^{i \theta}\right)^{\frac{1}{q}}}\right|\right)^{q} d \theta \leqslant \\
& \leqslant \sup _{0<r<1} \frac{1}{2 \pi} \int_{-\pi}^{\pi} \frac{c_{k}^{q}}{\mid 1-r_{k} r e^{i \theta \mid}} d \theta=\sup _{0<r<1} \frac{1}{2 \pi} \int_{-\pi}^{\pi} \frac{c_{k}^{q}}{\sqrt{1-2 r_{k} r \cos \theta+\left(r_{k} r\right)^{2}}} d \theta= \\
& =\sup _{0<r<1} \frac{1}{\pi} \int_{0}^{\pi} \frac{c_{k}^{q}}{\sqrt{\left(1-r_{k} r\right)^{2}+4 r_{k} r \sin ^{2}(\theta / 2)}} d \theta=\sup _{0<r<1} \frac{c_{k}^{q}}{\pi}\left(\int_{0}^{1-r_{k} r} \ldots+\int_{1-r_{k} r}^{\pi} \ldots\right) \leqslant \\
& \leqslant \sup _{0<r<1} \frac{c_{k}^{q}}{\pi}\left(\int_{0}^{1-r_{k} r} \frac{1}{\left(1-r_{k} r\right)} d \theta+\int_{1-r_{k} r}^{\pi} \frac{1}{\sqrt{\left(1-r_{k} r\right)^{2}\left(\frac{\theta}{\pi}\right)^{2}+4 r_{k} r\left(\frac{\theta}{\pi}\right)^{2}}} d \theta\right)= \\
& =\sup _{0<r<1} \frac{c_{k}^{q}}{\pi}\left(1+\int_{1-r_{k} r}^{\pi} \frac{d \theta}{\sqrt{\left(1+r_{k} r\right)^{2}\left(\frac{\theta}{\pi}\right)^{2}}}\right)=\sup _{0<r<1} \frac{c_{k}^{q}}{\pi}\left(1+\frac{\pi}{\left(1+r_{k} r\right)} \ln \frac{\pi}{1-r_{k} r}\right) \text {. }
\end{aligned}
$$

Taking into account the evident inequality $\ln x<x, \forall x>0$, and the condition (18) we conclude

$$
\sup _{0<r<1} \frac{1}{2 \pi} \int_{-\pi}^{\pi}\left(\ln ^{+}\left|f_{k}\left(r e^{i \theta}\right)\right|\right)^{q} d \theta \leqslant \sup _{0<r<1} \frac{c_{k}^{q}}{\pi}\left(1+\frac{\pi^{2}}{\left(1-\left(r_{k} r\right)^{2}\right)}\right)=\frac{c_{k}^{q}}{\pi}\left(1+\frac{\pi^{2}}{\left(1-r_{k}^{2}\right)}\right) \leqslant \gamma_{k}^{q} .
$$

We prove that $\left\{f_{k}\right\}$ is a bounded sequence in the class $\Pi_{q}$, i.e. we show that there exists a real number $0<\lambda<1$ such that for all natural numbers $k$ the inequality $\rho\left(\lambda f_{k}, 0\right)<\varepsilon$ is valid, where $\varepsilon$ is a fixed positive number (see $[12$, p. 31$])$. First, we prove that

$$
\ln (1+|\lambda||g|) \leqslant\left(\ln (1+|\lambda|)+\ln ^{+}|g|\right) .
$$

Indeed, if $|g| \leqslant 1$ then $|\lambda||g| \leqslant|\lambda|$, and the estimate (19) follows immediately.

If $|g| \geqslant 1$ then $\ln (1+|\lambda||g|) \leqslant \ln (|g|+|\lambda||g|) \leqslant \ln (1+|\lambda|)+\ln ^{+}|g|$.

Now we prove the inequality $\rho\left(\lambda f_{k}, 0\right)<\varepsilon$. Since $(a+b)^{q} \leqslant\left(a^{q}+b^{q}\right)$ for any $a>0, b>0$, $0<q<1$, using the estimate (19) we get

$$
\rho\left(\lambda f_{k}, 0\right)=\sup _{0<r<1} \frac{1}{2 \pi} \int_{-\pi}^{\pi} \ln ^{q}\left(1+\left|\lambda f_{k}\left(r e^{i \theta}\right)\right|\right) d \theta \leqslant \ln ^{q}(1+|\lambda|)+\left(\gamma_{k}\right)^{q} .
$$

Since $\gamma_{k}=o(1), k \rightarrow+\infty, \forall \varepsilon>0 \exists k_{0} \in \mathbb{N}: \forall k \geqslant k_{0}$ the following inequality holds: $\gamma_{k}<\sqrt[q]{\frac{\varepsilon}{2}}$. Choosing $\lambda_{k_{0}}$ so that $\ln \left(1+\left|\lambda_{k_{0}}\right|\right)<\sqrt[q]{\frac{\varepsilon}{2}}$, we see that starting from some number $k_{0}$ all elements of the sequence $\left\{f_{k}\right\}$ are contained in a ball of radius $\varepsilon$.

Since $\Pi_{q}$ is an $F$-space, for all numbers $k<k_{0}$ there exists a positive number $\lambda_{k}$ such that $\forall \lambda \in \mathbb{C},|\lambda| \leqslant \lambda_{k}$ the following inequality is valid: $\rho\left(\lambda f_{k}, 0\right)<\varepsilon$. Assuming $\lambda_{0}=$ $=\min \left(\lambda_{1}, \lambda_{2}, \ldots, \lambda_{k_{0}}\right)$, we obtain that for all $|\lambda| \leqslant \lambda_{0}$ the sequence $\left\{f_{k}\right\}$ is contained in a ball of radius $\varepsilon$, i.e. $\rho\left(\lambda f_{k}, 0\right)<\varepsilon$.

Owing to arbitrariness of the choice of $\varepsilon$, we conclude that $\left\{f_{k}\right\}$ is a bounded sequence in the class $\Pi_{q}$.

Since the sequence $\left\{f_{k}\right\}$ is bounded in $\Pi_{q}$, we get that the coefficient multiplier $\Lambda\left(f_{k}\right)$ is bounded in $X$. 
Let $X=H^{p}$. We have

$$
\left\|\Lambda\left(f_{k}\right)\right\|_{H^{p}} \leqslant C, C>0
$$

Fix a number $k \in \mathbb{N}$. If $f_{k}(z)=\sum_{n=0}^{+\infty} a_{n}^{(k)} z^{n} \in \Pi_{q}$, then $\Lambda\left(f_{k}\right)(z)=\sum_{n=0}^{+\infty} \lambda_{n} a_{n}^{(k)} z^{n} \in X$, therefore (see $[3$, c. 98])

$$
\begin{gathered}
\left|\lambda_{n} a_{n}^{(k)}\right| \leqslant c_{p}\left\|\Lambda\left(f_{k}\right)\right\|_{H^{p}} \cdot n^{\frac{1}{p}-1}, \text { for } \quad 0<p<1, \\
\left|\lambda_{n} a_{n}^{(k)}\right| \leqslant c_{p}\left\|\Lambda\left(f_{k}\right)\right\|_{H^{p}}, \text { for } \quad 1 \leqslant p \leqslant \infty,
\end{gathered}
$$

whence we have

$$
\begin{gathered}
\left|\lambda_{n} a_{n}^{(k)}\right| \leqslant C \cdot c_{p} \cdot n^{\frac{1}{p}-1}, \text { for } 0<p<1, \\
\left|\lambda_{n} a_{n}^{(k)}\right| \leqslant C \cdot c_{p}, \text { for } 1 \leqslant p \leqslant \infty
\end{gathered}
$$

where $c_{p}$ is a positive constant depending on $p$.

Since $f_{k}(z)=g\left(r_{k} z\right)$, we have $a_{n}^{(k)}=a_{n}\left(c_{k}\right) r_{k}^{n}$. According to Lemma 2.5,

$$
\left|a_{n}^{(k)}\right| \geqslant r_{k}^{n} \exp \left(c_{k}^{\frac{q}{q+1}} n^{\frac{1}{q+1}}\right) \text {. }
$$

Using the inequality (18), we have:

$$
\left|a_{k}^{(k)}\right| \geqslant\left(1-\frac{\pi^{2}}{k-1}\right)^{\frac{k}{2}} \exp \left(c_{k}^{\frac{q}{q+1}} k^{\frac{1}{q+1}}\right) .
$$

From (20), (22) we obtain the following estimate for $0<p<1$ :

$$
\left|\lambda_{k}\right| \leqslant C \cdot c_{p}^{\prime} \cdot\left(1-\frac{\pi^{2}}{k-1}\right)^{-\frac{k}{2}} \cdot k^{\frac{1}{p}-1} \cdot \exp \left(-c_{k}^{\frac{q}{q+1}} k^{\frac{1}{q+1}}\right)
$$

and applying now the estimate (16) we have:

$$
\left|\lambda_{k}\right| \leqslant \widetilde{C} \exp \left(-c_{k}^{\frac{q}{q+1}} k^{\frac{1}{q+1}}\right)
$$

From the inequality (23), applying Lemma 2.4, we conclude that the estimate (11) holds. Analogously, for $1 \leqslant p<+\infty$ from (21) and (23) we obtain the required estimate.

We prove the converse statement of Theorem 2.1. Let a sequence $\Lambda=\left\{\lambda_{k}\right\}$ satisfy the condition (11) of Theorem 2.1 and $f \in \Pi_{q}, f(z)=\sum_{k=0}^{+\infty} a_{k} z^{k}$. From Theorem 1.2 it follows that

$$
\left|a_{k}\right| \leqslant C_{1} \exp \left(\varepsilon_{k} k^{\frac{1}{q+1}}\right), \varepsilon_{k} \downarrow 0 .
$$

Choosing a number $k_{0}$ such that $\varepsilon_{k}<\frac{c}{2}$ for all $k \geqslant k_{0}$, we obtain:

$$
\left|\lambda_{k} a_{k}\right| \leqslant C_{2} \exp \left(-\frac{c}{2} k^{\frac{1}{q+1}}\right)
$$

Since the series $\sum_{k=0}^{+\infty} \exp \left(-\frac{c}{2} k^{\frac{1}{q+1}}\right)$ converges, $\Lambda(f)(z) \in X$ for any specified choice of class $X$. Theorem 2.1 is proved. 
Remark. Note that the method of the proof of Theorem 2.1 goes back to N. Yanagihara's work [19].

The immediate consequence of Theorem 2.1 is the statement that the estimates of Theorems 1.2 and 1.1 are unimprovable. The proof of this statement goes in the same manner as in the R. Meshtrovic's article (see [2, p. 152], Consequences 9.24, 9.26).

The author thanks Professor F.A.Shamoyan for carefully reading of the manuscript and helpful comments.

The work was financially supported by Russian Foundation for Fundamental Research, project 18-31-00180

\section{References}

[1] O.Blasco, M.Pavlovic, Coefficient multipliers on Banach spaces of analytic, 27(2011), no. 2, $415-447$.

[2] V.I.Gavrilov, A.V.Subbotin, D.A.Efimov, Boundary properties of analytic functions (further contribution), Moscow, Publishing House of the Moscow University, 2012 (in Russian).

[3] P.L.Duren, Theory of $H^{p}$ spaces, Pure and Appl. Math., vol. 38, Academic Press, NY, 1970.

[4] M.A.Evgrafov, The behavior of a power series for functions of the class $H_{\delta}$ on the boundary of the circle of convergence, Izv. AN SSSR. Ser. Mat., 16(1952), no. 5, 481-492 (in Russian).

[5] M.Jevtic, M.Pavlovic, Coefficient multipliers on spaces of analytic fuctions, Acta Sci. Math (Szeged), 64(1998), no. 3-4, 541-545.

[6] R.Mestrovic, A.V.Subbotin, Multipliers and linear functionals for Privalov spaces of holomorphic functions in the disc, Doklady Akademii Nauk, 365(1999), no. 4, 452-454 (in Russian).

[7] M.Pavlovic, Introduction to function spaces in a disk, Matematicki Institut SANU, Beograd, 2004.

[8] I.I.Privalov, Boundary properties of single-valued analytic functions, Moscow, Izd. Moscow Gos. Univ., 1941 (in Russian).

[9] I.I.Privalov, Boundary properties of analytic functions, M.-L., GITTL, 1950 (in Russian).

[10] E.G.Rodikova, On estimates of the expansion coefficients for certain classes of functions analytic in a disk, Proceedings of the VI Petrozavodsk International Conference «Complex Analysis and Applications», Petrozavodsk, PetrGU, 2012, 64-69 (in Russian).

[11] E.G.Rodikova, Factorization, characterization of zero sets, and questions of interpolation in weighted spaces of analytic functions, $\mathrm{PhD}$ thesis phis.-math. Sciences: 01.01.01, Bryansk, 2014 (in Russian).

[12] W.Rudin, Functional analysis, McGraw Hill, New York, 1973.

[13] R.F.Shamoyan, On the coefficient multipliers of Bloch and Hardy Spaces in a polydisk, Sib. Math. J., 43(2002), no. 1, 169-182. 
[14] F.A.Shamoyan, E.N.Shubabko, A Class of Functions Holomorphic in the Disk, Journal of Mathematical Sciences, 120(2004), no. 5, 1784-1790.

[15] F.A.Shamoyan, E.N.Shubabko, Introduction to the theory of weight $L^{p}$-classes of meromorphic functions, Bryansk, Group of Companies «Desyatochka», 2009 (in Russian).

[16] S.V.Shvedenko, Hardy classes and the spaces of analytic functions associated with them in the unit disc, polydisc, and ball, Itogi Nauki i Tekhniki. Ser. Mat. Anal., 23(1985), 3-124 (in Russian).

[17] S.V.Shvedenko, On the growth rate and Taylor coefficients of functions of the area Nevanlinna N class, Izv. vuzov. Mat., (1986), no. 6, 40-43 (in Russian).

[18] M.Stoll, Mean growth and Taylor coefficients of some topological algebras of analytic functions, Ann. Polon. Math., 35(1977), no. 2, 139-158.

[19] N.Yanagihara, Multipliers and linear functionals for the class $N^{+}$, Transactions of the Amer. Math. Soc., 180(1973), 449-461.

[20] N.Yanagihara, Mean growth and Taylor coefficients of some classes of functions, Ann. Polon. Math., 30(1974), 37-48.

\section{О коэффициентных мультипликаторах классов Привалова в круге}

Евгения Г. Родикова

Брянский государственный университет им. ак. И. Г. Петровского Бежицкая, 14, Брянск, 241036

Россия

В статъе получены точные оценки роста и коэффициентов разложения в ряд Тейлора функций из классов И.И. Привалова, полностъю описаны коэфбициентные мультипликаторы из класса Привалова в классы Харди.

Ключевые слова: класс Привалова, коэффициенты Тейлора, коэффиииентный мультипликатор, рост, аналитические функиии. 